\title{
Time Hopping Direct-Sequence CDMA for Asynchronous Transmitter-Only Sensors
}

\author{
Jianqiang Zeng, Hlaing Minn (Contact Author), and Lakshman S. Tamil \\ Department of Electrical Engineering, University of Texas at Dallas \\ Email: $\{$ jxz051000, hlaing.minn, tamil $\} @$ utdallas.edu
}

\begin{abstract}
This paper investigates transmission schemes for asynchronous transmitter-only wireless sensor nodes. We propose a time hopping DS-CDMA scheme by utilizing time hopping code design. Each sensor transmits only in pre-assigned active time slots and switches to sleep mode in idle time slots. By utilizing the proposed scheme, each sensor can always have several collision-free time slots. We also propose a new detector that has better BER performance than conventional matched filter (MF) detector by utilizing channel energy capturing and multipath diversity. We compare BER performance, power consumptions, and computational complexities of the proposed transmission scheme with conventional DS-CDMA scheme. The proposed scheme achieves significant energy and complexity savings while keeping similar BER performance with conventional DS-CDMA scheme.
\end{abstract}

\section{INTRODUCTION}

Wireless sensor networks were originally developed for military applications such as battlefield surveillance. They are now used in many civilian applications, including environment and habitat monitoring, health-care applications, home automation, and traffic control. Existing wireless sensor networks are based on regular communication systems (e.g., Bluetooth, Zigbee, UWB) where each sensor (sensor node) has transmission and reception capabilities. However, for some sensors the receiver portion may not be necessary. For example, some military applications may prefer maintaining secrecy of the location of the fusion node, enforcing no transmission from the fusion node to the sensors to avoid the risk of exposure of the fusion node. Exploiting transmitter-only sensors can provide significant reduction in complexity, cost, and energy consumption the much desirable features for wireless sensor networks. This will enable affordable energy-efficient wireless sensor network based services. Sensor nodes in environmental or health-care wireless monitoring applications may not require receiver, and large bandwidth may be available for these systems. Essential to this is the development of novel communication schemes for transmitter-only sensors which we will address in this paper.

The transmitter-only sensors mean there is no synchronization among sensors. The data rates of different sensors may also be different. This asynchronism in time and data rate among several sensors imposes an interesting communication design problem. There exist some works on synchronization in wireless sensor networks but they utilize two way links to perform synchronization (e.g., [1]). Asynchronous distributed sensors are typically used in location systems (e.g., [2]), however the signals from sensors are typically assumed to be received without any multi-user interference, and their main focus is how to efficiently fuse these signals in extracting the unknown parameters. The asynchronism issue was also addressed by means of medium access control (e.g., [3]), but this approach requires two way links. The synchronization of asynchronous DS-UWB systems was addressed in [4] by using two types of training signals. However, the users have both transmitter and receiver, and they receive common synchronization signal to estimate the timing. So their asynchronism are limited to small range. Different from the existing works, we will develop a communication scheme for asynchronous transmitter-only sensors. The sensors can transmit completely asynchronously. The communication schemes we present in this paper are based on DS-CDMA or a combination of DSCDMA and specifically designed time hopping codes. We also propose a new detector that has better BER performance than conventional matched filter (MF) detector by utilizing channel energy capturing and multipath diversity. We compare the energy consumption, computation complexity, and BER performance of the proposed time hopping DS-CDMA scheme with the conventional DS-CDMA scheme.

The rest of this paper is organized as follows. The system and signal models are described in Section II. The proposed time hopping transmission scheme is present in Section III. The energy consumption and complexity comparisons are discussed in Section IV and Section V, respectively. Simulation results and discussion are given in Section VI, and conclusions are drawn in Section VII.

\section{System Description AND Signal Model}

We consider an uplink asynchronous system with $K$ sensors and a sink node or fusion center. Each sensor has transmissiononly capability. The data signal of each sensor is defined as

$$
b_{k}(t)=\sum_{n=0}^{\infty} b_{k, n} p\left(t-n T_{b}\right)
$$

where $p(t)$ is the rectangular pulse shape, and $T_{b}$ is the bit duration. $b_{k, n}$ is the DPSK modulated data defined as

$$
b_{k, n}=e^{j \theta_{n}}=e^{j\left(\pi a_{k, n}+\theta_{k, n-1}\right)},
$$


where $a_{k, n}$ is the $n$th information bit of $k$ th sensor, and the initial phase $\theta_{k,-1}=0$.

Each data signal $b_{k}(t)$ is spread by the spreading code $c_{k}(t)$. The spreading code is defined as

$$
c_{k}(t)=\sum_{m=0}^{N_{c}-1} c_{k, l} \psi\left(t-m T_{c}\right),
$$

where $c_{k, m}$ is $m$ th chip of the spreading code for the sensor $k, \psi(t)$ is rectangular chip waveform, $T_{c}$ is the chip duration and $N_{c}=\frac{T_{b}}{T_{c}}$ is the processing gain. The spreading code is normalized such that $\int_{0}^{T_{b}}\left|c_{k}(t)\right|^{2} d t=1$. To avoid the intersymbol interference (ISI), we insert guard interval into the beginning and the end of the spreading code. Guard interval is preferred over cyclic prefix (CP) due to its energy saving.

The equivalent low-pass transmitted signal of sensor $k$ is given by

$$
s_{k}(t)=A b_{k}(t) c_{k}(t)
$$

where $A$ is the amplitude scaling factor.

The time domain signal samples of different sensors are transmitted through independent multipath fading channels. The sample-spaced channel impulse response for the $k$ th sensor can be given by

$$
h_{k}(t)=\sum_{l=0}^{L-1} h_{k, l}(t) \delta\left(t-l T_{s}\right)
$$

where $h_{k, l}(t)$ and $l T_{s}$ are the complex gain and the delay of the $l$ th channel tap, $T_{s}$ is the sampling period, and $L$ is the number of sample-spaced channel taps.

The received signal from $K$ sensors is given by

$$
r(t)=\sum_{k=1}^{K} s_{k}\left(t-\tau_{k}\right) * h_{k}(t)+w(t)
$$

where $\tau_{k}$ is the unknown delay corresponding to the $k$ th sensor, and $w(t)$ is the additive white Gaussian noise (AWGN) process.

The received signal is sampled at $M_{s}=\frac{T_{c}}{T_{s}}$ times the chip rate, so the discrete time sequence is given by

$$
r(i)=\sum_{k=1}^{K} s_{k}\left(i T_{s}-d_{k}\right) * h_{k}(i)+w(i)
$$

where $d_{k}=\tau_{k} / T_{s}$, and $w(i)$ is a complex Gaussian noise sample with zero mean and variance $\sigma_{n}^{2}=E\left[|\omega(i)|^{2}\right]$.

The receiver performs sync (packet) detection and timing synchronization for each sensor. Each sensor transmits preamble consisting of $N_{t}$ identical parts with possible sign change as $[ \pm \mathbf{x}, \pm \mathbf{x}, \ldots, \pm \mathbf{x}]$, where $\mathbf{x}$ represents a training bit spread by the same spreading code. The best training symbol pattern can be obtained by computer search (The details are refereed to [5]). The received signal is cross-correlated with $M_{s}$ times oversampled training sequences. The sync detection metric is given by

$$
\lambda_{k}(d)=\left|\sum_{i=0}^{N_{t} N_{c} M_{s}-1} r(i+d) \cdot v_{k}^{*}(i)\right|^{2}
$$

where $\left\{v_{k}(i): i=0,1, \ldots, N_{t} N_{c} M_{s}-1\right\}$ is the $M_{s}$ oversampled training sequence of the $k$ th sensor. The presence of packet is determined by the sync detection metric and an associated threshold $\eta$, i.e., $\lambda_{k}\left(d_{k \text {,sync }}-i\right) \leq \eta$ with possible integer $i$ and $\lambda_{k}\left(d_{k \text {,sync }}\right)>\eta$, where $d_{k \text {,sync }}$ is the packet detected timing point of the $k$ th sensor.

After obtaining $d_{k, \text { sync }}$, the coarse timing offset estimate of the $k$ th sensor is given by

$$
\hat{d}_{k}^{c}=\underset{d \in\left[d_{k, \mathrm{sync}}, d_{k, \mathrm{sync}}+W-1\right]}{\operatorname{argmax}}\left\{\lambda_{k}(d)\right\},
$$

where $W$ is the search window length.

In an AWGN channel, the mean of the proposed timing metric trajectory peak is at the exact timing point, but in multipath channels it would be shifted due to the channel dispersion. The coarse-timing estimate can be fine-tuned by capturing the channel energy as

$$
\hat{d}_{k}=\underset{d}{\operatorname{argmax}}\left\{\sum_{d=\hat{d}_{k}^{c}-1}^{\hat{d}_{k}^{c}+L-2} \lambda_{k}(d)\right\} .
$$

Note that the knowledge of $L$ or its approximate value can be obtained from the application environment.

After obtaining the timing estimates, the received signal synchronized to the sensor $k$ is despread by multiplying with the corresponding spreading code as

$$
\tilde{b}_{k, n}=\sum_{i=0}^{N_{c}-1} r\left(i \cdot M_{s}+\left(M_{s}-1\right)+\hat{d}_{k}+n N_{c} M_{s}\right) c_{k}(i) \text {. }
$$

The conventional data detection is performed as

$$
\hat{a}_{k, n}= \begin{cases}1, & \text { if } \operatorname{Re}\left\{\tilde{b}_{k, n} \cdot \tilde{b}_{k, n-1}^{*}\right\}<0 \\ 0, & \text { if } \operatorname{Re}\left\{\tilde{b}_{k, n} \cdot \tilde{b}_{k, n-1}^{*}\right\}>0\end{cases}
$$

In order to improve the detection performance, we modify the detector structure as

$$
\hat{a}_{k, n}= \begin{cases}1, & \text { if } \operatorname{Re}\left\{\sum_{l=-1}^{L-2}\left(\bar{b}_{k, n}(l) \cdot \bar{b}_{k, n-1}^{*}(l)\right)\right\}<0 \\ 0, & \text { if } \operatorname{Re}\left\{\sum_{l=-1}^{L-2}\left(\bar{b}_{k, n}(l) \cdot \bar{b}_{k, n-1}^{*}(l)\right)\right\}>0\end{cases}
$$

where $\bar{b}_{k, n}(l)$ is given by

$\bar{b}_{k, n}(l)=\sum_{i=0}^{N_{c}-1} r\left(i \cdot M_{s}+\left(M_{s}-1\right)+\hat{d}_{k}+n N_{c} M_{s}+l\right) c_{k}(i)$

This modified detector can better capture the channel energy and exploit multipath diversity to improve the detection performance.

The average SNR is defined as

$$
\mathrm{SNR}_{\mathrm{avg}}=\frac{\sum_{i=0}^{M_{s} N_{c}-1}\left|s_{k}\left(i T_{s}\right)\right|^{2}}{M_{s} N_{c} \sigma_{n}^{2}}
$$




\section{Proposed Time Hopping DS-CDMA TRANSMISSION SCHEME}

In the conventional DS-CDMA transmission scheme, all sensors transmit the signal during the whole time slots. The probability of the collision is high. And the transceiver is always active, so there always exists circuit energy consumption in this transmission scheme. In the short ranging communication scenario which is considered in this paper, the circuit energy consumption is comparable to or even larger than the transmission energy. Hence the battery energy can be significantly saved by reducing the circuit energy consumption. This motivates us to design a novel communication scheme that can reduce the energy consumption and the computation complexity for the considered scenario. The energy consumption comparison and computation complexity comparison are discussed in Section IV and Section V.

In the proposed transmission scheme, each sensor is assigned to a time hopping code. The sensors transmit only on those time slots assigned to them and switch to the sleep mode during the rest of time slots. On each assigned time slot, the sensor transmits a spreading code, i.e., each element of the time hopping code is spread with a spreading code. For preamble, each element of the time hopping code is spread with a different spread code.

Denote the indices of active time slots and idle time slots of the $k$ th sensor as $\mathcal{T}_{k}$ and $\mathcal{I}_{k}$, respectively. $\mathcal{T}_{k} \cap \mathcal{I}_{k}=\oslash$ and $\mathcal{T}_{k} \cup \mathcal{I}_{k}=\{1,2, \ldots, N\} .\left[\mathcal{T}_{k}\right]_{l}$ is defined as the $l$ th element in $\mathcal{T}_{k} . N$ is the number of total time slots per information symbol. $M$ is the number of the active time slots which each of the $K$ sensors is assigned to. The time hopping code designs are based on the following rule that any arbitrarily shifted time hopping code has at most one same time slot index as any other arbitrarily shifted time hopping code. This ensures each sensor has at least $M-(K-1)$ non-overlapped time slots. We present two time hopping code designs in the following.

The proposed time hopping code design A (Design A): The time hopping code design $\mathrm{A}$ is based on an index set $\mathcal{S}$ of $M K$ elements with distinctive spacings. The distinctive spacing is defined as $[\mathcal{S}]_{i_{1}}-[\mathcal{S}]_{j_{1}}=[\mathcal{S}]_{i_{2}}-[\mathcal{S}]_{j_{2}}$ only if $i_{1}=i_{2}$ and $j_{1}=j_{2}$. In design A, $\mathcal{T}_{k}$ are obtained by dividing the set $\mathcal{S}$ into $K$ disjoint subsets. To maintain at least $M-$ $(K-1)$ non-overlapped time slots in asynchronous scenarios, distinctive spacings need to be preserved for modulo $N$, i.e., $\left([\mathcal{S}]_{i_{1}}-[\mathcal{S}]_{j_{1}}\right)_{N}=\left([\mathcal{S}]_{i_{2}}-[\mathcal{S}]_{j_{2}}\right)_{N}$ only if $i_{1}=i_{2}$ and $j_{1}=j_{2}$, where $(\cdot)_{N}$ is the module $N$ operation.

The characteristics of distinctive spacing based time hopping code design are given as follows.

1) $\mathcal{T}_{k} \cap \mathcal{T}_{j}=\oslash$ when $k \neq j$,

2) $\left[\mathcal{T}_{k}\right]_{l}-\left[\mathcal{T}_{j}\right]_{i}$ are distinctive for $l \neq i$,

3) $M \geq K$

4) $N=2 \cdot \max \left\{\mathcal{T}_{k}: k=1, \ldots, K\right\}$.

The time hopping code design example is given below. Suppose a sensor network consists of $K=3$ sensors, and each sensor is assigned to $M=4$ time slots. The time hopping codes based on the above design are given as follows: $\mathcal{T}_{1}=\{1,2,4,8\}, \mathcal{T}_{2}=\{13,21,31,45\}$ and $\mathcal{T}_{3}=\{66,91,117,145\}$. They can also be given as: $\mathcal{T}_{1}=\{1,8,31,91\}, \mathcal{T}_{2}=\{2,13,45,117\}$ and $\mathcal{T}_{3}=$ $\{4,21,66,145\}$. The number of total time slots is $N=$ $2 \cdot 145=290$. The time hopping code can be expressed as

$$
t h_{k}(n)= \begin{cases}1, & n \in \mathcal{T}_{k} \\ 0, & n \in \mathcal{I}_{k}\end{cases}
$$

Each sensor transmits short spreading codes, e.g. Gold sequence, in its active time slots and switches to the sleep mode in idle time slots.

The proposed time hopping code design B (Design B): When the number of sensors increases, the total number of time slots required to satisfy distinctive spacing property becomes large. To reduce the required total time slots, a modified version of the time hopping code design is presented as follows. We relax the 1 st and the 2nd characteristics of design A. The mutual spacings of the time hopping codes do not need to be distinctive. And each code can start with the same time slot index. The only requirement we need is the arbitrarily shifted $\mathcal{T}_{k}, k=\{1, \ldots, K\}$ has at most one same time slot index as any arbitrary shifted $\mathcal{T}_{j}, j \neq k$. This requirement ensures each sensor has at least $M-(K-1)$ nonoverlapped time slots. An algorithm satisfying this requirement is described as follows.

1) All codes start from 1 ,

2) $\mathcal{T}_{1}$ is assigned to consecutive time indices, e.g., $\{1,2, \ldots, M\}$

3) $\mathcal{T}_{k}$ is $\{1,(M+k-2)+1,2(M+k-2)+1, \ldots,(M-$ 1) $(M+k-2)+1\}$. During the code element candidate search, each candidate needs to be verified if it satisfies the timing hopping code design requirement described above or not. If not, the candidate value is increased by 1 till it satisfies the requirement.

4) $N=2 \cdot \max \left\{\mathcal{T}_{k}: k=1, \ldots, K\right\}$.

A design example of this modified algorithm is given as follows. $\mathcal{T}_{1}=\{1,2,3,4\}, \mathcal{T}_{2}=\{1,5,9,13\}$ and $\mathcal{T}_{3}=$ $\{1,6,11,16\}$. The total number of time slots required is reduced to $N=2 \cdot 16=32$.

\section{ENERGY CONSUMPTION COMPARISONS}

Transmission energy is the major energy consumption in long range communications. However in short range communications such as personal or body area networks, circuit energy consumption can be comparable to or even larger than the transmission energy [6]. In this section, we compare the total energy consumption of both transmission schemes.

Assume the average time required to transmit one bit is $T^{1}$, and the transceiver circuitry can work on multi-mode basis, i.e., when the sensor is transmitting signal, all circuits work in the active mode, the transceiver switches to the sleep mode when there is no signal to transmit, and there is a transient

\footnotetext{
${ }^{1}$ For proposed time hopping DS-CDMA, one bit duration consists of $N$ time slots, and length- $N_{s c}$ spreading code is transmitted on each active time slot.
} 
mode when the transceiver switches from the sleep mode to the active mode. The time duration of the active mode, the sleep mode and the transient mode are denoted by $T_{\mathrm{on}}, T_{\mathrm{sp}}$ and $T_{\mathrm{tr}}$, respectively. For the conventional DS-CDMA transmission scheme, the transceiver remains in the active mode during the whole time $T$, i.e., $T_{\text {on }}=T, T_{\mathrm{sp}}=T_{\mathrm{tr}}=0$. So the total energy consumption is given as

$$
E_{\mathrm{CV}}=P_{\mathrm{ont}} \cdot T
$$

where $P_{\text {ont }}$ is transmitter power consumption for the active mode. $P_{\text {ont }}$ that consists of three parts is defined as [6]

$$
P_{\mathrm{ont}}=P_{\mathrm{t}}+P_{\mathrm{amp}}+P_{\mathrm{ct}},
$$

where $P_{\mathrm{t}}$ is the transmission power, $P_{\mathrm{amp}}$ is the amplifier power consumption, and $P_{\mathrm{ct}}$ is the circuit power consumption. The power amplifier (PA) power consumption can be modeled as $P_{\text {amp }}=\alpha P_{\mathrm{t}}$, where $\alpha$ is depends on both the drain efficiency of the RF PA and the peak-to-average ratio (PAR).

For the proposed time hopping DS-CDMA transmission scheme, we have $T=T_{\mathrm{on}}+T_{\mathrm{sp}}+T_{\mathrm{tr}}$. The total energy consumption is given as

$$
E_{\mathrm{TH}}=P_{\mathrm{ont}} \cdot T_{\mathrm{on}}+P_{\mathrm{sp}} \cdot T_{\mathrm{sp}}+P_{\mathrm{tr}} \cdot T_{\mathrm{tr}},
$$

where $P_{\mathrm{sp}}$ and $P_{\mathrm{tr}}$ are the power consumption of the sleep mode and the transient mode, respectively.

Substituting equation (18) into (17) and (19), we have

$$
\begin{gathered}
E_{\mathrm{CV}}=(1+\alpha) P_{\mathrm{t}} \cdot T+P_{\mathrm{ct}} \cdot T \\
E_{\mathrm{TH}}=(1+\alpha) P_{\mathrm{t}} \cdot T_{\mathrm{on}}+P_{\mathrm{ct}} \cdot T_{\mathrm{on}}+P_{\mathrm{sp}} \cdot T_{\mathrm{sp}}+P_{\mathrm{tr}} \cdot T_{\mathrm{tr}}
\end{gathered}
$$

The power consumption in the sleep mode is much smaller than the power consumption in the active mode, and hence it can be neglected, i.e., $P_{\mathrm{sp}}=0$. From [6], the power consumption in the transient mode only needs to include the power consumption of the frequency synthesizers $P_{\text {syn }}$, and hence $P_{\mathrm{tr}} \approx P_{\mathrm{syn}}$. To ensure fair comparison, we keep the same active mode transmission energy for both schemes, i.e., $(1+\alpha) P_{\mathrm{t} 1} \cdot T=(1+\alpha) P_{\mathrm{t} 2} \cdot T_{\mathrm{on}}$, where $P_{\mathrm{t} 1}$ and $P_{\mathrm{t} 2}$ are the transmission power of the conventional scheme and the proposed scheme, respectively. So the main energy consumption difference of these two schemes comes from the circuit energy consumption. Because $T_{\text {on }}$ and $T_{\mathrm{tr}}$ are much smaller than $T$, and $P_{\text {syn }}$ is generally smaller than $P_{\text {ct }}$, the energy saving can be approximated as

$$
E_{\text {save }}=E_{\mathrm{CV}}-E_{\mathrm{TH}} \approx P_{\mathrm{ct}} \cdot\left(T-T_{\mathrm{on}}\right) .
$$

For the simulation parameters we used in the Section VI, $T_{\text {on }}$ is only $\frac{6}{84}$ of $T$, so large energy saving can be achieved by exploiting the proposed time hopping transmission scheme.

\section{COMPuTATION COMPLEXITY COMPARISONS}

For the proposed time hopping DS-CDMA scheme, a short spreading code is used in each assigned active time slots. Denote $N_{s c}$ as the length of the short spreading code. To ensure fair comparison, we keep the same processing gain for both transmission schemes. So the length of the long spreading code used in the conventional DS-CDMA scheme is given as $N_{c}=N \cdot N_{s c}$, where $N$ is the number of total time slots in one bit duration for the proposed time hopping DS-CDMA scheme. For each possible candidate timing point, the timing synchronization metric requires $N_{t} N_{c} M_{s}+1$ complex multiplications, $N_{t} N_{c} M_{s}-1$ complex additions, 1 real addition, and 1 compare operation for the conventional scheme. For the proposed scheme, it requires $N_{t} M N_{s c} M_{s}+1$ complex multiplications, and $N_{t} M N_{s c} M_{s}-1$ complex additions, 1 real addition, and 1 compare operation.

For data detection in the conventional scheme, the despread operation requires $N_{c}$ complex multiplications, and $N_{c}-1$ complex additions for the MF detection scheme. $(L-1) N_{c}$ complex multiplications, and $(L-1) N_{c}-1$ complex additions are needed for the modified detection scheme. For the proposed transmission scheme, the despread operation requires $M N_{s c}$ complex multiplications, and $M N_{s c}-1$ complex additions for the MF detection scheme. $(L-1) M N_{s c}$ complex multiplications, and $(L-1) M N_{s c}-1$ complex additions are needed for the modified detection scheme.

Because $N_{c}=N \cdot N_{s c}$ and $M \ll N$, the proposed time hopping DS-CDMA scheme has much lower computation complexity compared to the conventional DS-CDMA scheme.

\section{Simulation RESUltS AND DISCUSSIONS}

We consider an uplink asynchronous DS-CDMA system with $K=4$ sensor nodes. $M=6$ time slots are assigned to each sensor for the proposed scheme. The indices of the active time slots based on the time hopping code design $\mathrm{B}$ are given in the Table I. So the number of total time slots in one bit duration is $N=2 \cdot 42=84$. The short spreading code used in the proposed scheme is Gold sequence with length 31 . In each active time slot, length $L_{z p}=8$ zeroes are added to the beginning and the end of Gold sequence. So the $N_{s c}=31+2 \cdot 8=47$ for the proposed scheme. For the conventional scheme, the same length $L_{z p}=8$ zeroes are added to the beginning and the end of PN sequence. So the length of the long PN spreading code used in the conventional scheme is $N_{c}=N \cdot N_{s c}=84 \cdot 47-2 \cdot 8=3932$. The bit energy of both schemes are normalized to 1 . In our simulation, we use burst mode transmission where the preamble (training symbols) is preceded by noise samples and followed by data symbols. The training symbol pattern used is $[-\mathbf{x},+\mathbf{x},-\mathbf{x},-\mathbf{x}]$. Each frame consists of 4 training bits and 32 data bits. The Rayleigh fading channel with $L=4$ sample-spaced taps is used in the simulation. The channel has an exponential power delay profile with $3 \mathrm{~dB}$ per tap decay rate.

Fig. 1 shows the BER performance of the conventional DSCDMA scheme and the proposed time hopping DS-CDMA scheme with perfect timing offset knowledge. The sampling interval of the transmitted signal, channel, and the received signal is $\frac{1}{4}$ of the chip duration. We compare the BER performance of two detectors, one is conventional matched filter (MF) detector, the other one is the modified detector (MD) which is given in (13). The proposed scheme has the 
TABLE I

THE INDicES OF ACTIVE TIME SLOTS

\begin{tabular}{|c|c|}
\hline Sensor & Indices of Active Time Slots \\
\hline 1 & {$[1,2,3,4,5,6]$} \\
\hline 2 & {$[1,7,13,19,25,31]$} \\
\hline 3 & {$[1,8,15,22,29,36]$} \\
\hline 4 & {$[1,9,17,26,34,42]$} \\
\hline
\end{tabular}

TABLE II

COMPUTATION COMPLEXITIES COMPARISONS

\begin{tabular}{||c|c|c|c|c||}
\hline \multirow{2}{*}{} & \multicolumn{2}{|c|}{ Timing Metric Calculation } & \multicolumn{2}{c||}{ Despread Operation } \\
\cline { 2 - 5 } & Cmp. mul. & Cmp. add. & Cmp. mul. & Cmp. add. \\
\hline PN & 62,913 & 62,911 & 3932 & 3931 \\
\hline TH & 2,977 & 2,975 & 186 & 185 \\
\hline
\end{tabular}

same BER performance as the conventional scheme for both detectors. The modified detector has lower BER than the conventional MF detector because the modified detector takes channel dispersion into account. It gives about $7 \mathrm{~dB}$ SNR advantage at an uncoded BER of $10^{-2}$ over the conventional detector.

Fig. 2 shows the BER comparisons of both schemes for two detectors with perfect timing knowledge. The sampling interval of the transmitted signal and the channel is $\frac{1}{4}$ of the chip duration. But the sampling interval of the received signal is $\frac{1}{2}$ of the chip duration. So there are mismatches between the actual timing points and the estimated timing points due to sampling rate mismatch. The BER degradation of the proposed detector is negligible in Fig. 2 compared with Fig. 1. Hence the receiver sampling rate of 2 times chip rate is sufficient for asynchronous scenarios.

Fig. 3 shows the BER performance of the conventional DSCDMA scheme and the proposed time hopping DS-CDMA scheme with sync detection threshold $\eta=3$ and timing offset estimation. The timing estimator given in (10) is used in the simulation. The proposed scheme has the same BER performance as the conventional scheme for all considered scenarios.

Fig. 4 shows the packet loss rate versus sync detection threshold. The higher threshold has higher packet loss rate as expected. At the threshold $\eta=3$ which we set for $E_{b} / N_{0}=20 \mathrm{~dB}$, the packet loss rate is about $3 \%$. Note that average SNR is only $-16 \mathrm{~dB}$ for $\mathrm{E}_{\mathrm{b}} / \mathrm{N}_{0}=20 \mathrm{~dB}$.

The computational complexity comparisons using the simulation parameters are shown in Table II. For simplicity, this table only shows the operations of 1 candidate timing metric calculation and 1 bit despread operation for data detection. The proposed transmission scheme requires about $\frac{1}{21}$ of the complexity of the conventional scheme.

\section{CONCLUSIONS}

We investigate transmission schemes for asynchronous transmitter-only wireless sensor networks. We propose a novel time hopping DS-CDMA scheme which achieves significant power saving and computational complexity reduction over conventional DS-CDMA scheme while keeping the same BER performance. The advantages of the proposed scheme can

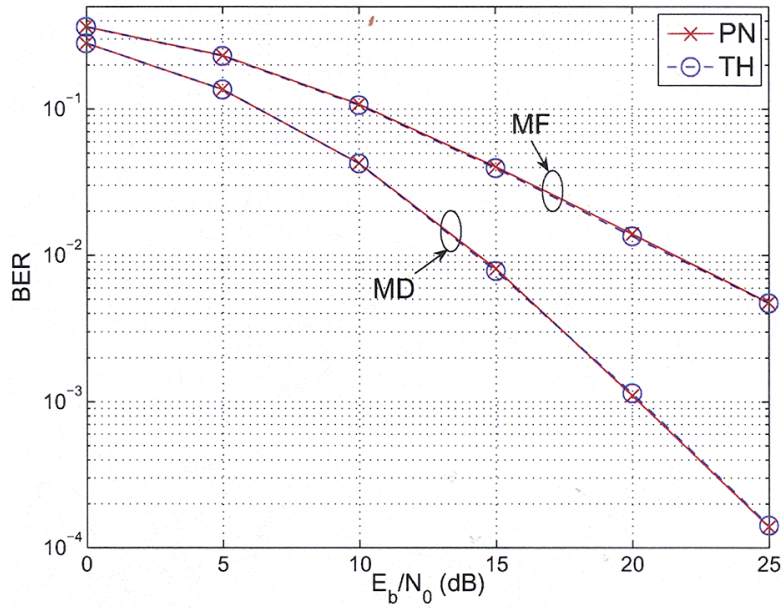

Fig. 1. BER performance comparisons with perfect timing

be well appreciated in developing affordable energy-efficient wireless sensor networks based services, e.g., sensor nodes in environmental or health-care wireless monitoring applications. We also propose a new detector by utilizing channel energy capturing and multipath diversity. The proposed transmission scheme achieves substantial complexity saving (about 20 times) over the conventional transmission scheme. The proposed detector provides much better BER performance (about $7 \mathrm{~dB}$ SNR advantage at BER of $10^{-2}$ ) than the conventional detector.

\section{REFERENCES}

[1] M. Nordman, W. Kozlowski, and O. Vahamaki, "A method for synchronizing low cost energy aware sensors used in industrial process monitoring," in Proc. 27th Annual Conference of the IEEE Industrial Electronics Society IECON '01, vol. 1, 2001, pp. 100-106 vol.1.

[2] T. Li, A. Ekpenyong, and Y.-F. Huang, "A location system using asynchronous distributed sensors," in Proc. IEEE Asia-Pacific Conference on Circuits and Systems, vol. 1, 2004, pp. xvii-xvii.

[3] C. Busch, M. Magdon-Ismail, F. Sivrikaya, and B. Yener, "Contention free MAC protocols for asynchronous wireless sensor networks," Journal of Distributed Computing, to appear.

[4] M. Hernandez and R. Kohno, "Code acquisition and timing-frame synchronization for asynchronous DS-UWB transmission systems," in Proc. IEEE 2006 International Conference on Ultra-Wideband, 2006, pp. 1318.

[5] H. Minn, V. Bhargava, and K. Letaief, "A robust timing and frequency synchronization for OFDM systems," IEEE Trans. Wireless Commun., vol. 2, no. 4, pp. 822-839, Jul. 2003.

[6] S. Cui, A. Goldsmith, and A. Bahai, "Energy-constrained modulation optimization," IEEE Trans. Wireless Commun., vol. 4, no. 5, pp. 23492360, 2005. 


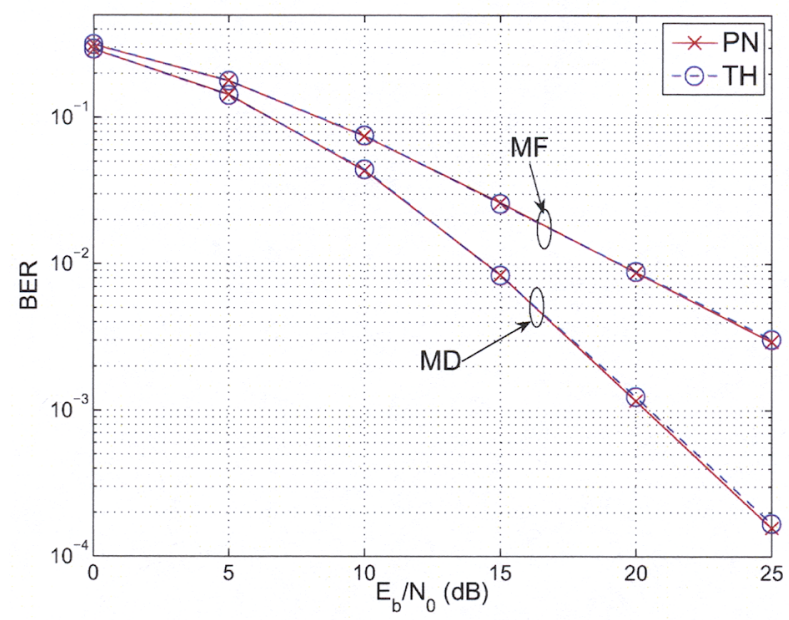

Fig. 2. BER performance comparisons with perfect timing

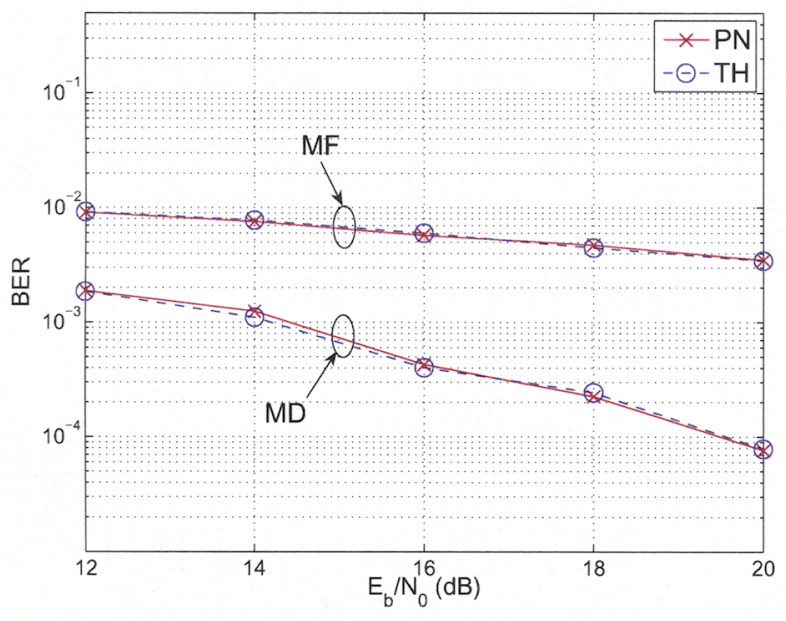

Fig. 3. BER performance comparisons with timing estimation

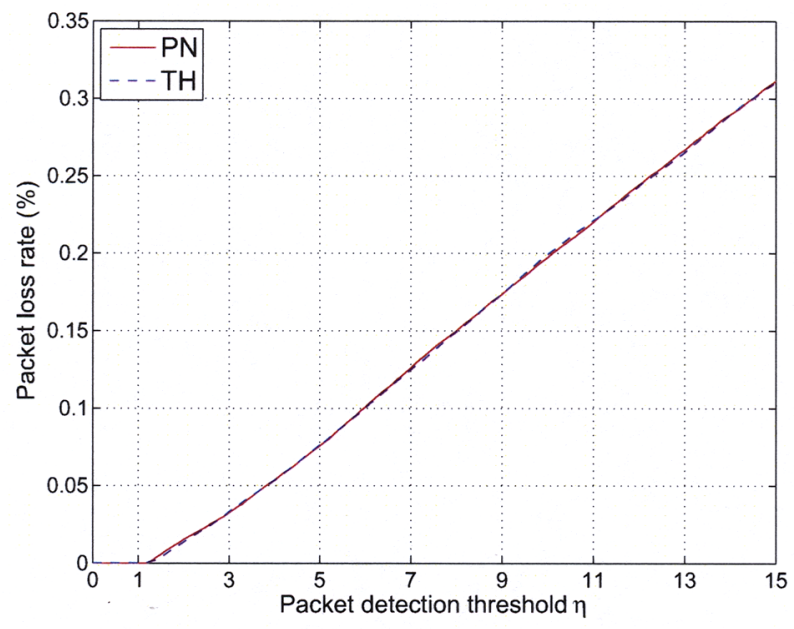

Fig. 4. Packet loss rate vs threshold 